\title{
The effect of unsaturated fatty acid and triglyceride oil addition on the mechanical and antibacterial properties of acrylic bone cements
}

\author{
Cecilia Persson $^{1 *}$, Elise Robert ${ }^{1}$, Elin Carlsson ${ }^{1}$, Céline Robo $^{1}$, Alejandro López ${ }^{1}$, \\ Maria Godoy-Gallardo ${ }^{2}$, Maria-Pau Ginebra ${ }^{2,3}$, Håkan Engqvist ${ }^{1}$
}

${ }^{1}$ Division of Applied Materials Science, Department of Engineering Sciences, The Ångström Laboratory, Uppsala University, Uppsala, Sweden

${ }^{2}$ Biomaterials, Biomechanics and Tissue Engineering Group, Department of Materials Science and Metallurgy, Technical University of Catalonia (UPC), Barcelona, Spain

${ }^{3}$ Biomedical Research Networking Center in Bioengineering, Biomaterials and Nanomedicine (CIBER-BBN), Spain

\author{
*Correspondence: \\ E-mail: cecilia.persson@angstrom.uu.se \\ Telephone: +46-184717911 \\ Fax: +46-18-4713572
}

\begin{abstract}
Acrylic bone cements have an elastic modulus several times higher than the surrounding trabecular bone. This has been hypothesized to contribute to certain clinical complications. There are indications that the addition of specific fatty acids and triglyceride oils may reduce the elastic modulus of these types of cements. Some of these additives also appear to have inherent antibiotic properties, although this has never been evaluated in bone cements. In this study, several types of fatty acids and triglyceride oils were evaluated for use in acrylic bone cements. Their mechanical properties were evaluated under uniaxial compression testing and selected cements were then further characterized in terms of microstructure, handling and antibacterial properties using scanning electron microscopy, polymerization temperature measurements, agar diffusion tests and bactericidal activity assays of cement extracts. It was found that any of the evaluated fatty acids or triglyceride oils could be used to tailor the stiffness of acrylic bone cements, although at varying concentrations, which also depended on the type of commercial base cement used. In particular, the addition
\end{abstract}


of very small amounts of linoleic acid $(<2.0 \mathrm{wt} \%)$ resulted in Young's moduli and compressive strengths in the range of human trabecular bone, while maintaining a similar setting time. Further, the addition of $12.6 \mathrm{wt} \%$ ricinoleic acid to Osteopal V cement was found to have a significant antibacterial effect, inhibiting growth of Staphylococcus aureus in an agar diffusion test as well as demonstrating $100 \%$ bactericidal activity against the same strain.

Keywords: PMMA, bone cement, low-modulus, mechanical, antibacterial, fatty acid, triglyceride oil 


\section{Introduction}

Acrylic bone cements, mainly based on poly(methyl methacrylate) (PMMA), have been used in orthopedics since the 1960's for joint prosthesis fixation and since the 1980's for vertebroplasty, and are still extensively used. ${ }^{1-3}$ These cements are readily available as a system consisting of a liquid and a powder to be mixed and applied in a liquid form as an injectable material or in a more viscous form as a paste. They can also be used in their set state as e.g. beads or spacers for local treatment of infection. ${ }^{4}$ A basic formulation consists of a) a powder, which is composed of prepolymerized polymer beads mixed with a radical initiator, typically benzoyl peroxide, and a radiopacifier, typically barium sulphate or zirconium dioxide; and b) a liquid, which is composed of acrylic monomer, and a peroxide decomposer, typically $\mathrm{N}, \mathrm{N}-$ dimethyl- $p$-toluidine. Some formulations contain other additives such as dyes, ${ }^{5}$ used to differentiate the cement from the bone in the case of open surgery or explantation; bioactive fillers, ${ }^{6}$ used with the aim of enhancing bone integration; additional radiopacifier, for enhanced visibility in minimally invasive applications, ${ }^{7,8}$ and antibiotics, to prevent or treat infection. ${ }^{4,7}$ Although these additives are available in commercial formulations, cements are some times modified directly in the operating theater with some of these additives, e.g. through the addition of extra radiopacifier to cements intended for prosthesis fixation, ${ }^{7,8}$ or through the addition of antibiotics. ${ }^{4,7}$ Besides the above modifications, experimental formulations containing e.g. plasticizers have been investigated in order to reduce the stiffness of the cements. ${ }^{9}$ This is because the excessive stiffness of acrylic bone cements in comparison to the surrounding trabecular bone may contribute to biomechanically related complications, mainly noted after vertebroplasty. ${ }^{10-13}$ In fact, most commercial acrylic bone cements have an elastic modulus in the range of $1700-3700 \mathrm{MPa},{ }^{14}, 15$ while the elastic modulus of vertebral trabecular bone is typically in the ranges of $10-750 \mathrm{MPa},{ }^{16-18}$ encompassing osteoporotic to healthy bone. New fractures occurring after vertebroplasty may partly be due to the underlying disease, but some studies have reported a larger amount of new fractures next to the vertebrae treated with PMMA. ${ }^{19-21}$ The maximum filling approach, in which as much cement as possible is injected into the vertebra, has been suggested to have a particularly detrimental effect. ${ }^{22,23}$. However, the stiffness and strength of vertebrae have been found to be only weakly correlated with the volume fill ${ }^{24-26}$, suggesting that even relatively small amounts of 
high-stiffness PMMA cements may induce a large increase in stiffness. Several experimental studies have therefore focused on developing novel PMMA-based cements with a lower elastic modulus by using additives such as hydrogels ${ }^{27,28}$ or lactam plasticizers. ${ }^{29}$ Even though these attempts have proved successful in terms of tailoring the mechanical properties of the cements, none of these cements are commercially available. One reason for this could be that an excessive particle release has been found when aqueous phases are used in PMMA to obtain a reduced modulus $^{30}$.

Unsaturated fatty acids, which esterified to glycerol form triglycerides, are natural compounds that have recently been given more attention in chemical processes because of their potential for chemical modifications due to unsaturations and carboxyl groups in their structure. ${ }^{31}$ Different approaches to incorporate compounds from this family into bone cements have been attempted. For instance, Vázquez et al. ${ }^{32}$ used aromatic amines as well as acrylic monomers, both derived from oleic acid, as components to improve bone cements. The authors reported similar compressive strength and a reduction of $62 \%$ in the Young's modulus when a modified liquid phase containing $2.57 \mathrm{wt} \%$ 4-N,N-dimethylaminobenzyl oleate was used. ${ }^{32}$ Lam et al. ${ }^{33}$ modified bone cements with strontium-substituted hydroxyapatite-nanoparticles and linoleic acid, and reported a reduction of $15 \%$ in compressive strength and $57 \%$ in the Young's modulus when 15 vol\% linoleic acid was incorporated into the liquid phase. ${ }^{33}$ In previous work, we added castor oil to commercially available PMMA and found that $12 \mathrm{wt} \%$ castor oil decreased the strength and the Young's modulus by $83 \%$ and $70 \%,{ }^{34}$ respectively. However, the castor oil appeared to interfere with the setting reaction, reducing the cell viability in a worst-case scenario. ${ }^{34}$ Although further, preliminary testing has suggested that a modification of the monomer-to-additive ratio could improve biocompatibility, the possibility of using another type of fatty acid or derivative thereof, which requires lower concentrations, remains an interesting opportunity. However, there is no systematic study available comparing different types of modifiers in PMMA bone cement. One aim of this study was therefore to screen different compounds in the fatty acid/triglyceride oil family to select the most suitable additive to control the mechanical properties of commercial bone cements for vertebroplasty. Furthermore, since these compounds could remain unreacted and provide an increase in intermolecular free volume, there may be an additional positive effect in terms of the drug release of the cement. PMMA cements alone have a limited 
drug delivery capacity, with the main delivery stemming from the surface of the cement, which means an excessive amount of drug has to be incorporated into the cement. A large quantity of the drug remains inside the cement and slowly leaks out, possibly causing increased bacterial resistance in the case of antibiotics. ${ }^{35}$ Unreacted additive molecules may provide additional paths for drug release and hence an enhanced drug delivery from the PMMA cement, requiring less antibiotic addition. Furthermore, some of the compounds of interest have demonstrated inherent antibiotic properties and could therefore enhance the effect of an incorporated antibiotic. ${ }^{36,37}$ However, to the authors' knowledge, no report is available on the antibacterial effect of combining a fatty acid with an antibiotic. A second aim of this study was hence to evaluate the antibacterial properties of modified cements, with and without antibiotics. To meet the aims of the study, various commonly found fatty acids and triglyceride oils, namely, ricinoleic acid, methyl linoleate, linoleic acid, tung oil, linseed oil, and castor oil were incorporated into two types of commercial bone cements for screening. The mechanical properties were evaluated under uniaxial compression and selected cements were then further characterized in terms of microstructure, handling, antibacterial properties and monomer release using scanning electron microscopy (SEM), polymerization temperature measurements, agar diffusion tests and bactericidal activity assays as well as monomer content of cement extracts.

\section{Materials and methods}

\subsection{Material preparation}

All chemicals were purchased from Sigma-Aldrich (St. Louis, MO, USA) unless otherwise specified. A cement intended for vertebroplasty, Osteopal V (OP; Heraeus Medical GmbH, Hanau, Germany) was used as one base cement. Osteopal V has a liquid to powder ratio of $0.385 \mathrm{ml} / \mathrm{g}$ and the powder phase contains $54.6 \mathrm{wt} \%$ poly(methyl acrylate-co-methyl methacrylate), $45.0 \mathrm{wt} \%$ zirconium dioxide, $0.4 \mathrm{wt} \%$ benzoyl peroxide, and chlorophyll VIII. The monomer phase contains $92 \mathrm{wt} \%$ methyl methacrylate, $2 \mathrm{wt} \% \mathrm{~N}, \mathrm{~N}$-dimethyl- $p$-toluidine and $6 \mathrm{wt} \%$ other additives including chlorophyll VIII and hydroquinone. A cement for prosthesis fixation, Simplex P (SP; Stryker Corporation, Kalamazoo, MI, USA) was also used as base cement, however for mechanical testing only, to confirm the concept for these types of cements. Simplex P has a liquid to powder ratio of $0.5 \mathrm{ml} / \mathrm{g}$ and the powder phase contains 73.7 
$\mathrm{wt} \%$ poly(methyl methacrylate-co-styrene), $15 \mathrm{wt} \%$ poly(methyl methacrylate), 10 $\mathrm{wt} \%$ barium sulphate, $1.7 \mathrm{wt} \%$ benzoyl peroxide. The monomer phase contains 97.5 wt $\%$ methyl methacrylate and $2.5 \mathrm{wt} \% \mathrm{~N}, \mathrm{~N}$-dimethyl- $p$-toluidine. The following compounds in the fatty acid/triglyceride oil family were tested as additives: linoleic acid (LA; $\geq 99 \%$ ), ricinoleic acid (RA; 80\%), methyl linoleate (ML; $\geq 99 \%$ ), tung oil (TO), linseed oil (LO), and castor oil (CO). Modified cements were prepared by first removing $54 \%$ of the additive volume from the monomer phase. The additive was then added to the monomer and the modified monomer phase was homogenized using a Vortex-Genie 2 mixer (Scientific Industries, Bohemia, NY, USA) prior to cement preparation. The bone cements were prepared by adding the monomer phase to the powder phase inside $50 \mathrm{~mL}$ Falcon tubes, and then mixed for 30 seconds using a capvibrator (Ivoclar Vivadent AB, Solna, Sweden).

\subsection{Mechanical testing}

Specimens of $\varnothing=6 \mathrm{~mm}$ and $\mathrm{h}=12 \mathrm{~mm}$ were tested after $24 \mathrm{~h}$ setting in air at room

temperature. ${ }^{38}$ Uniaxial compression tests were performed under displacement control at $6 \mathrm{~mm} / \mathrm{min}$ using an AGS-X materials testing machine (Shimadzu, Kyoto, Japan). The Young's modulus $(E)$ and compressive strength $\left(\sigma_{\mathrm{c}}\right)$ (yield stress at $2 \%$ offset or upper yield point, whichever occurred first ${ }^{38}$ ) were determined from the stress-strain data. Since different additives affected the properties to different extent, various concentrations were tested until cements with a combination of a Young's modulus below $750 \mathrm{MPa}$ and a strength above 10MPa were obtained, since such Young's modulus is in the upper range of elastic moduli of vertebral trabecular bone, 16, 18 and the strength corresponds to the highest values reported for vertebral trabecular bone ${ }^{17}$ (although mixed with femoral specimens - other highest values reported for vertebral trabecular bone lie at approx. $5 \mathrm{MPa}{ }^{18}$ ). Based on the results, a few compositions were selected for further characterization, as further detailed in section 3.1 .

\subsection{Microstructure}

The microstructure of the cements was examined by scanning electron microscopy (SEM). The images were acquired on a LEO 1550 scanning electron microscope (Carl Zeiss AG, Oberkochen, Germany) operated at an acceleration voltage of $2.00 \mathrm{kV}$, 
with a secondary electron detector for topographic contrast, and at a working distance of $10 \mathrm{~mm}$. Prior to SEM imaging, the samples were polished down to 1200 grit and sputter-coated with palladium. The samples were prepared $24 \mathrm{~h}$ prior to polishing and then stored under vacuum overnight prior to coating.

\subsection{Handling properties}

The maximum polymerization temperature $\left(\mathrm{T}_{\max }\right)$ and the setting time $\left(\mathrm{t}_{\mathrm{set}}{ }_{\mathrm{ing}}\right)$, defined as the time taken to reach a temperature midway between ambient and maximum temperature, were determined according to the standard procedure described in ISO 5833, with four replicates per group. ${ }^{38}$ The glass transition temperature of the cements was assessed through differential scanning calorimetry using a DSC Q1000 (TA Instruments, United States). A heat/cool/heat ramp experiment was performed using a heating rate of $10^{\circ} \mathrm{C} / \mathrm{min}$ up to $200^{\circ} \mathrm{C}$ and a cooling rate of $20^{\circ} \mathrm{C} / \mathrm{min}$, down to $-80^{\circ} \mathrm{C}$. The glass transition temperature was determined from the second heat ramp.

\subsection{Antimicrobial properties}

Vancomycin was added to selected cements in order to evaluate the antibacterial properties of the modified cements as well as the effect of combining a fatty acid or its derivative with an antibiotic. Vancomycin was chosen as it is commonly used in PMMA bone cement for revision arthroplasty. ${ }^{39,40}$

The antibacterial activity of the samples was tested using the gram-positive bacterium Staphylococcus aureus (Culture Collection, University of Göterborg, Sweden). S.aureus was grown and maintained in Brain Heart Infusion (BHI) broth (Scharlau, Spain). The culture was incubated overnight at $37^{\circ} \mathrm{C}$ before each assay and the assays were performed in static conditions due to the short testing time.

Agar diffusion test: The relative antibacterial activity of selected formulations with and without antibiotic (2.4 wt\% of powder $\left.{ }^{39}\right)$ was determined through an agar diffusion test. The test was performed by spreading $200 \mu \mathrm{L}$ of bacterial suspension $\left(1 \cdot 10^{8}\right.$ colony forming units $(\mathrm{CFU}) / \mathrm{mL}$, O.D. of $0.2 \pm 0.01$ at $\left.600 \mathrm{~nm}\right)$ homogenously on every BHI agar plate, previously prepared with the appropriate culture media containing $1.5 \%$ bacteriological agar (Scharlau, Spain). Disc-shaped specimens $(\varnothing=7$ $\mathrm{mm} ; \mathrm{h}=3.5 \mathrm{~mm}$ ) of cements were prepared and four equidistant cavities of the same size were punched in the agar and the specimens placed inside, after $1 \mathrm{~h}$ of setting in 
air at room temperature. Images of the specimens were taken with a digital camera after incubation for 24 hours at $37^{\circ} \mathrm{C}$. The inhibition zone size was calculated as the mean difference between the inhibition zone diameter and the cement-filled cavity diameter. Six replicates were performed per formulation.

Bactericidal assay: The antimicrobial activity of cement extracts was assessed using disc-shaped specimens $(\varnothing=7.5 \mathrm{~mm} ; \mathrm{h}=2 \mathrm{~mm})$ of cements. Extracts were prepared by soaking the cements, after $1 \mathrm{~h}$ of setting in air at room temperature, in phosphate buffered saline (PBS) solution according to the ISO 10993 standard at a surface-toPBS medium of $3 \mathrm{~cm}^{2} / \mathrm{mL}$ for 24 hours at $37^{\circ} \mathrm{C}$. ${ }^{41}$ Staphylococcus aureus $\left(2 \times 10^{5}\right.$ $\mathrm{CFU} / \mathrm{mL}$ ) was incubated in the cement extracts for 2 hours at $37^{\circ} \mathrm{C} .100 \mu \mathrm{L}$ aliquots from these extracts were subsequently spread on agar plates, and the number of surviving colonies counted after incubation for 24 hours at $37^{\circ} \mathrm{C}$. The percentage of surviving colonies was calculated with respect to the surviving colonies in the positive control (PBS only). Four replicates per formulation were performed.

\subsection{Monomer release}

Extracts were prepared after $1 \mathrm{~h}$ of setting in air at room temperature, using six specimens $(\varnothing=7.7 \mathrm{~mm}, h=3.5 \mathrm{~mm})$ per group. Extractions were done for each group by submerging all 6 specimens in $3.55 \mathrm{~mL}$ PBS for $24 \mathrm{~h}$ at $37^{\circ} \mathrm{C}$, similarly to the antibacterial tests. Afterwards, $2 \mathrm{~mL}$ aliquots from each solution were introduced in a head space vial and closed hermetically. The vials were incubated at $80^{\circ} \mathrm{C}$ during 30 min. The analysis was performed by gas chromatography-mass spectrometry (HSGC/MS), by injecting $1 \mathrm{~mL}$ of the vapor phase through a special syringe kept at $85^{\circ} \mathrm{C}$. A Trace GC Gas Chromatograph with Triplus head space injector coupled to a DSQII mass spectrometer (ThermoFisher Scientific, Waltham, MA) was used. A ZB-624 column $(60 \mathrm{~m} \times 0.32 \mathrm{~mm} \times 1.8 \mathrm{~m})$ with a helium flow of $1.8 \mathrm{~mL} / \mathrm{min}$ was used for separation. The oven temperature programme consisted of 2 min hold at $60^{\circ} \mathrm{C}$, followed by $8^{\circ} \mathrm{C} / \mathrm{min}$ ramp to $220^{\circ} \mathrm{C}$ and a $5 \mathrm{~min}$ hold at $220^{\circ} \mathrm{C}$. The temperature of the injector, interface, and ionization source was set at 220,260 , and $200^{\circ} \mathrm{C}$, respectively. 


\subsection{Statistical Analysis}

IBM SPSS Statistics v. 21 (IBM, Chicago, IL, USA) was used to perform analyses of variance (ANOVA) followed by Scheffe's post-hoc test in order to evaluate statistical differences between groups. When homogeneity of variance could not be confirmed (using Levene's test), Welch's robust test of equality of means was used together with Tamhane's post-hoc test. A significance level of $\alpha=0.05$ was used.

\section{Results}

\subsection{Mechanical properties}

The properties of the base (control) cements in which the additives were evaluated are summarized in Table 1. Table 2 shows the mechanical properties of Simplex P and Osteopal V modified with fatty acids: ricinoleic acid, linoleic acid methyl ester (methyl linoleate) and linoleic acid. Linoleic acid as well as its methyl ester had a more pronounced effect on the Young's modulus of both Simplex P and Osteopal V compared to ricinoleic acid. Table 3 shows the mechanical properties of Simplex $\mathrm{P}$ and Osteopal V modified with triglyceride oils: linseed oil, tung oil and castor oil. Linseed oil and tung oil had a more pronounced effect on the Young's modulus than castor oil. In general, lower amounts of additive were required to reach similar mechanical properties for Osteopal V than for Simplex P.

Two fatty acids (linoleic acid and ricinoleic acid) and one triglyceride oil (castor oil) were selected for further testing with Osteopal V: more ricinoleic acid than lineoleic acid or methyl linoleate was needed for the same effect on the properties, while the effect of methyl linoleate was similar to that of linoleic acid; further, castor oil contains mainly ricinoleic acid but also linoleic acid and no methyl linoleate. Regression analysis was performed on the Young's modulus and ultimate strength results of the cements containing the selected additives, and cements predicted to have a similar modulus and fulfilling the criteria of a Young's modulus below $750 \mathrm{MPa}$ and a strength above $10 \mathrm{MPa}$ were further characterized. These were Osteopal V cements containing $1.0 \mathrm{wt} \%$ linoleic acid, $12.6 \mathrm{wt} \%$ ricinoleic acid and $12.3 \mathrm{wt} \%$ castor oil, hereinafter referred to as OP-1.0\%LA, OP-12.6\%RA and OP-12.3\%CO. Their mechanical properties, with and without antibiotic, were confirmed according to the same testing protocol as before (see section 2.2), and these results are shown in Table 4. Cements containing Vancomycin are designated with VA, i.e. Osteopal V cement 
modified with $1.0 \mathrm{wt} \% \mathrm{LA}$ and $2.4 \mathrm{wt} \%$ Vancomycin (of powder) is named OP1.0\%LA-VA. OP and OP-VA had significantly higher elastic moduli and compressive strengths than the other cements $(\mathrm{p}<0.001)$. No other statistically significant differences were found, i.e. no statistically significant difference was found between OP and OP-VA $(\mathrm{p}=0.1)$, nor between OP-1.0\%LA, OP-12.6\%RA and OP-12.3\%CO as well as their corresponding Vancomycin-containing formulations $(\mathrm{p}>0.05)$.

\subsection{Microstructure}

The surface microstructure of Osteopal V and its modified low-modulus formulations was assessed through scanning electron microscopy (Figure 1). Osteopal V modified with linoleic acid (Figure 1B) exhibited a similar microstructure to that of unmodified Osteopal V (Figure 1A), displaying a dense, highly polymerized material, with no major features. On the other hand, low-modulus Osteopal V modified with ricinoleic acid and castor oil (Figures $1 \mathrm{C}$ and D, respectively) exhibited a more irregular porous surface where the spherical polymer beads $(20-90 \mu \mathrm{m})$ as well as the irregular zirconia particles (used as radiopacifier) were distinguishable.

\subsection{Handling properties}

The handling properties of the selected cements are shown in Table 5. The setting times of cements containing ricinoleic acid and castor oil were significantly lower than the control $(\mathrm{p} \leq 0.001)$, but there was no significant difference between the two (p>0.05). All low-modulus formulations gave significantly lower polymerization temperatures than the unmodified cement $(\mathrm{p}<0.001)$, but no difference was found between the modified cements $(\mathrm{p}>0.05)$.

\subsection{Antimicrobial properties}

The inhibitory zones resulting from the agar diffusion test are shown in Figure 2A. Unmodified Osteopal V showed virtually no inhibitory zone. A clear inhibitory effect was however observed for all the formulations when Vancomycin was added $(p \leq 0.01)$. An inhibitory effect could also be seen for the formulations without antibiotic but containing other additives, although this effect was statistically significant for ricinoleic acid only $(\mathrm{p}<0.001$, in comparison to unmodified Osteopal V). 
The results for the bactericidal activity of the extracts are shown in Figure $2 \mathrm{~B}$ as percentage of viable bacteria in comparison to PBS only. Unmodified Osteopal V was significantly different only to cements containing ricinoleic acid, with or without antibiotic $(\mathrm{p}=0.02)$, which both showed the highest bactericidal activity (no viable bacteria). No difference was found between groups with and without antibiotic, except for cements containing linoleic acid $(\mathrm{p}=0.01)$.

\subsection{Monomer release}

Chromatography-mass spectrometry (HS-GC/MS) gave a concentration of MMA in the extracts of $1.40,2.13,0.75$ and $0.96 \mathrm{~g} / 1$ for OP, OP-1.0\%LA, OP-12.6\%RA and $\mathrm{OP}-12.3 \% \mathrm{CO}$, respectively.

\section{Discussion}

In this study different fatty acids and triglyceride oils were tested as potential additives to acrylic bone cements with two purposes: A) to tailor the Young's modulus towards values closer to those of human trabecular bone; B) to achieve enhanced antibacterial properties of the cements. To screen the applicability of these compounds in the clinical setting we chose two base cements, Osteopal V, intended for vertebroplasty, and Simplex P, intended for prosthesis fixation. The results for the mechanical properties clearly indicated that among the tested fatty acids, linoleic acid and its methyl ester had the largest effect on the final values since lower additive concentrations were required to achieve properties similar to human trabecular bone (Table 2). The reason for this could be the increased availability of the end hydrogen atom for abstraction in combination with the polyunsaturation of linoleic acid and its methyl ester in comparison to ricinoleic acid. The level of unsaturation of a fatty acid or a triglyceride is commonly measured in terms of the iodine value (the mass of iodine in grams that is consumed by $100 \mathrm{~g}$ of a substance), which is 181.0 and $85.1 \mathrm{mg}$ for linoleic and ricinoleic acid, respectively ${ }^{31}$ indicating that a higher iodine value increases the reactivity of the fatty acid within the bone cement. Methacrylation of the polyunsaturated fatty acids can occur via chain transfer. ${ }^{42}$ Previous studies on a similar type of reaction have suggested that the methyl methacrylate (MMA) radicals may graft by abstraction of the alpha-hydrogen in the fatty acid. ${ }^{42}$ Thereby, fatty acids reduce the modulus of the cement through grafting and formation of stable radicals that slow down the polymerization and shorten the chains. ${ }^{42}$ Also, the 
presence of these large molecules increases the free volume and facilitates the relative movement of the polymer chains, thereby decreasing the glass transition temperature and lowering the elastic modulus of the material, as seen in Table 5. The results also indicated that among the tested triglyceride oils, linseed and tung oil had the largest effect on the final mechanical properties (Table 3). The major components of linseed, tung, and castor oil, are linolenic acid (55\%), $\alpha$-Eleostearic acid (82.0\%), and ricinoleic acid $(87.5 \%)$, respectively. ${ }^{31}$ The iodine values for triglycerides from these fatty acids are $261.6 \mathrm{mg}$ for linolenic and $\alpha$-Eleostearic acid and $81.6 \mathrm{mg}$ for ricinoleic acid. This indicates that, also for the triglyceride oils, the higher efficacy in decreasing the mechanical properties stems from the reactivity of the additive.

Larger amounts of additives were generally required in Simplex P compared to Osteopal V to reach similar moduli. This followed from the relative amounts of cement components (see section 2.1): in order to reach a similar additive to monomer ratio, a larger weight percentage of the total cement was needed in Simplex P. Interestingly, a much lower vol\% (approximately $3 \mathrm{vol} \%$ compared to $15 \mathrm{vol} \%$ ) of linoleic acid was needed to achieve a similar reduction in modulus in the current study compared to the study by Lam et al. ${ }^{43}$ However, in the study by Lam et al., the cements also contained $20 \mathrm{wt} \%$ (of powder) linoleic-acid functionalized strontium substituted hydroxyapatite particles, which hence appeared to limit the effect of the added linoleic acid, possibly due to the hypothesized formation of a copolymer on the particle-cement interface. ${ }^{43}$

The setting time decreased for the cements containing ricinoleic acid and castor oil compared to the control, while the cement containing linoleic acid gave no significant difference. The use of fatty acids or triglyceride oils also had a positive effect on the peak polymerization temperature, which was reduced by almost $30^{\circ} \mathrm{C}$. A reduction in peak polymerization temperature could be beneficial from a clinical perspective, as high polymerization temperatures may have negative effects on surrounding tissues. ${ }^{44,45}$ It can be noted, however, that the amounts used to measure the setting time and maximum temperature according to the standard ${ }^{38}$ are much larger than those used in e.g. vertebroplasty. The additives reduced the glass transition temperature of the modified cements, which could be due to shorter polymer chains besides increased interchain spacing as explained above. The decrease in setting time, polymerization temperature and glass transition temperature of cements containing ricinoleic acid and castor oil could also be related to the smaller amount of monomer that was available 
to react in these formulations (see section 2.1). Since the activator was present in excess in the liquid phase, approximately the same number of reactions could be started by the initiator from the powder phase, giving a shorter setting time and shorter chains. In fact, the SEM images (Figure 1) indicated that the morphology of these cements was not homogeneous, being affected by the presence of a nonpolymerizing phase. Conversely, the cement containing linoleic acid appeared homogeneous and its setting time was not significantly affected.

The agar diffusion test showed that the antibiotic Vancomycin was released from all the materials creating an inhibitory zone that prevented the growth of Staphylococcus aureus (Figure 2A). No significant improvement in inhibitory effect was detected as a result of any of the additives, despite the apparently more open structure of the ricinoleic acid and castor oil containing cements. However, the test confirmed that the materials containing fatty acids or a triglyceride oil had a certain inhibitory effect even without added antibiotic. ${ }^{36,37}$ Regarding the bactericidal activity of cement extracts (Figure 2B), a difference due to the addition of antibiotic was found for cements containing linoleic acid only, likely due to relatively large variances in some cases together with the limited number of specimens (3-4 per group). In fact, in a previous study, where a similar amount of Vancomycin in another commercial formulation (Cemex XL, Tecres) was used, ${ }^{39}$ a bactericidal activity was found without other additives than the antibiotic. However, much larger cement disks were used $(\varnothing=15 \mathrm{~mm}$; $\mathrm{h}=10 \mathrm{~mm}$ compared to our $\varnothing=7.5 \mathrm{~mm}$; $\mathrm{h}=2 \mathrm{~mm}$ ), together with a lower seeding density $\left(1 \cdot 10^{5} \mathrm{CFU} / \mathrm{ml}\right.$ in comparison to our $\left.2 \cdot 10^{5} \mathrm{CFU} / \mathrm{ml}\right)$. Similarly to the agar test, the additives used in the present study appeared to give a bactericidal effect of their own, but a significant difference to the control was detected only for cements containing ricinoleic acid, which showed $100 \%$ bactericidal activity, i.e. no viable bacteria was detected, regardless of the use of antibiotic. Although, to the best of the authors knowledge, there is no data available on the antibacterial effects of ricinoleic acid itself, some of its derivatives have been found to have antibacterial properties $^{46}$. Residual monomer may have toxic effects ${ }^{47}$, however the monomer release study confirmed that the bactericidal effect of ricinoleic acid-containing cements was not likely to be due to an increase in monomer release from these cements. In fact, the ricinoleic acid- as well as the castor oil-containing cements gave a lower monomer release than the control, possibly due to the removal of monomer 
from the original composition during preparation of these cements (see section 2.1) and the faster setting. Any possible negative biological effect of the ricinoleic acidcontaining cements however needs to be evaluated in a future study. The addition of linoleic acid, which entailed removal of much less monomer than for the other modified cements, gave a higher monomer release than the control, possibly due to the reduced polymerization rate, as discussed above. However, preliminary data indicates that although linoleic acid- and castor oil-containing cements give a lower cell viability for osteoblast-like Saos-2 cells in contact with undiluted extracts, dilutions of as little as 4-10 times give viabilities not significantly different to the control (data not shown). Future work should include in vivo evaluation of the cements' biocompatibility as well as further studies elucidating the possible antibacterial effect of the ricinoleic acid.

Further, it should be noted that, while in the current study a lower modulus was aimed for, the modifications also entailed a lower strength. A strength of above 10MPa was set as the limit, in line with literature values for vertebral trabecular bone ${ }^{17,18}$. However, while this may be adequate for the vertebroplasty application, the ISO standard for acrylic cements for prosthesis fixation cites a minimum strength of $70 \mathrm{MPa}^{38}$. Hence, while the use of the additives could be useful for lowering the modulus of vertebroplastic cements, and/or achieving antibacterial properties without the need for an additional antibiotic, the use of these cements in other applications may be restricted to limited or non load-bearing situations, such as beads for the local treatment of infection ${ }^{48}$.

In summary, PMMA based cements with a lower elastic modulus as well as improved antibacterial properties were produced using fatty acids and triglyceride oils. Whereas low-modulus cements modified with small amounts of linoleic acid gave a similar morphology to unmodified cements, low-modulus cements containing much higher amounts of ricinoleic acid and castor oil gave a more porous structure. Ricinoleic acid appeared to have inherent antibacterial properties.

\section{Conclusion}

The addition of fatty acids or triglyceride oils is a simple and effective method to modify the properties of existing commercial bone cements or to design new bone cements with bone-compliant mechanical properties and antibacterial properties. Specifically, very small amounts of linoleic acid $(<2.0 \mathrm{wt} \%)$ can be used to obtain 
Young's moduli and compressive strengths in the range of human trabecular bone. Further, the addition of $12.6 \mathrm{wt} \%$ ricinoleic acid to Osteopal V cement was found to have a significant antibacterial effect, inhibiting growth of Staphylococcus aureus in an agar diffusion test as well as demonstrating $100 \%$ bactericidal activity against the same strain. This suggests that ricinoleic acid could replace commonly used antibiotics in some instances, e.g. in pre-polymerized beads; whereas if only a reduction in modulus is desired, linoleic acid could be used, e.g. in vertebroplasty.

\section{Acknowledgements}

N/A

\section{Declaration of conflicting interests}

One of the materials evaluated in this study has been described in patent application nr PCT/SE2014/050429, where co-authors Cecilia Persson and Alejandro López are co-inventors. Co-authors Elise Robert, Elin Carlsson, Maria Godoy-Gallardo, MariaPau Ginebra and Håkan Engqvist have no conflicts of interest.

\section{Funding}

This work was supported by the EU [grant number FP7-PEOPLE-2010-268134], VINNOVA [VINNMER project 2010-02073], the Swedish Foundation for International Cooperation in Research and Higher Education (STINT) [grant number IG2011-2047], and the Spanish Government [Project MAT2012-38438-C03-01, cofunded by the EU through European Regional Development Funds]. Support for the research of MPG was received by the Generalitat de Catalunya [ "ICREA Academia" prize for excellence in research].

\section{References}

1. Charnley J. Anchorage of the Femoral Head Prosthesis to the Shaft of the Femur. Journal of Bone \& Joint Surgery, British Volume. 1960; 42-B: 28-30.

2. Galibert P, Deramond H, Rosat P, et al. Preliminary note on the treatment of vertebral angioma by percutaneous acrylic vertebroplasty. Neurochirurgie. 1987; 33: 166-168.

3. O'Brien JP, Sims JTandEvans AJ. Vertebroplasty in Patients with Severe Vertebral Compression Fractures: A Technical Report. American Journal of Neuroradiology. 2000; 21: 1555-1558. 
4. Magnan B, Bondi M, Maluta T, et al. Acrylic bone cement: current concept review. Musculoskelet Surg. 2013; 97: 93-100.

5. Webb JCJandSpencer RF. The role of polymethylmethacrylate bone cement in modern orthopaedic surgery. Journal of Bone \& Joint Surgery, British Volume. 2007; 89-B: 851-857.

6. Hernández L, Parra J, Vázquez B, et al. Injectable acrylic bone cements for vertebroplasty based on a radiopaque hydroxyapatite. Bioactivity and biocompatibility. Journal of Biomedical Materials Research Part B: Applied Biomaterials. 2009; 88B: 103-114.

7. Fourney DR, Schomer DF, Nader R, et al. Percutaneous vertebroplasty and kyphoplasty for painful vertebral body fractures in cancer patients. J Neurosurg. 2003; 98: 21-30.

8. Lewis G. Injectable bone cements for use in vertebroplasty and kyphoplasty: state-of-the-art review. J Biomed Mater Res B Appl Biomater. 2006; 76: 456468.

9. Boger A, Wheeler K, Montali A, et al. NMP-modified PMMA bone cement with adapted mechanical and hardening properties for the use in cancellous bone augmentation. J Biomed Mater Res B Appl Biomater. 2009; 90: 760-766.

10. Grados F, Depriester C, Cayrolle G, et al. Long-term observations of vertebral osteoporotic fractures treated by percutaneous vertebroplasty. Rheumatology. 2000; 39: 1410-1414.

11. Uppin AA, Hirsch JA, Centenera LV, et al. Occurrence of new vertebral body fracture after percutaneous vertebroplasty in patients with osteoporosis1. Radiology. 2003; 226: 119-124.

12. Trout AT, Kallmes DFandKaufmann TJ. New Fractures after Vertebroplasty: Adjacent Fractures Occur Significantly Sooner. AJNR Am J Neuroradiol. 2006; 27: 217-223.

13. Chen W-J, Kao Y-H, Yang S-C, et al. Impact of Cement Leakage Into Disks on the Development of Adjacent Vertebral Compression Fractures. Journal of Spinal Disorders \& Techniques. 2010; 23: 35-39.

14. Kurtz SM, Villarraga ML, Zhao K, et al. Static and fatigue mechanical behavior of bone cement with elevated barium sulfate content for treatment of vertebral compression fractures. Biomaterials. 2005; 26: 3699-3712.

15. Hernandez L, Muñoz ME, Goñi I, et al. New injectable and radiopaque antibiotic loaded acrylic bone cements. Journal of Biomedical Materials Research Part B: Applied Biomaterials. 2008; 87B: 312-320.

16. Morgan EF, Bayraktar HHandKeaveny TM. Trabecular bone modulus-density relationships depend on anatomic site. J Biomech. 2003; 36: 897-904.

17. Nazarian A, von Stechow D, Zurakowski D, et al. Bone volume fraction explains the variation in strength and stiffness of cancellous bone affected by metastatic cancer and osteoporosis. Calcif Tissue Int. 2008; 83: 368-379.

18. Kopperdahl DLandKeaveny TM. Yield strain behavior of trabecular bone. $J$ Biomech. 1998; 31: 601-608.

19. Burton AW, Mendoza T, Gebhardt R, et al. Vertebral compression fracture treatment with vertebroplasty and kyphoplasty: experience in 407 patients with 1,156 fractures in a tertiary cancer center. Pain Med. 2011; 12: 1750-1757.

20. Nieuwenhuijse MJ, Putter H, van Erkel AR, et al. New vertebral fractures after percutaneous vertebroplasty for painful osteoporotic vertebral compression fractures: a clustered analysis and the relevance of intradiskal cement leakage. Radiology. 2013; 266: 862-870. 
21. Trout AT, Kallmes DF, Layton KF, et al. Vertebral endplate fractures: an indicator of the abnormal forces generated in the spine after vertebroplasty. $J$ Bone Miner Res. 2006; 21: 1797-1802.

22. Berlemann U, Ferguson SJ, Nolte L-P, et al. Adjacent vertebral failure after vertebroplasty: a biomechanical investigation. The Journal of Bone \& Joint Surgery (British Volume). 2002; 84-B: 748-752.

23. Baroud G, Nemes J, Heini P, et al. Load shift of the intervertebral disc after a vertebroplasty: a finite-element study. European Spine Journal. 2003; 12: 421426.

24. Dean JR, Ison KTandGishen P. The strengthening effect of percutaneous vertebroplasty. Clin Radiol. 2000; 55: 471-476.

25. Liebschner MAK, Rosenberg WSandKeaveny TM. Effects of Bone Cement Volume and Distribution on Vertebral Stiffness After Vertebroplasty. Spine. 2001; 26:--.

26. Reidy D, Ahn H, Mousavi $\mathrm{P}$, et al. A biomechanical analysis of intravertebral pressures during vertebroplasty of cadaveric spines with and without simulated metastases. Spine. 2003; 28: 1534-1539.

27. Boger A, Bisig A, Bohner M, et al. Variation of the mechanical properties of PMMA to suit osteoporotic cancellous bone. Journal of Biomaterials Science, Polymer Edition. 2008; 19: 1125-1142.

28. Boger A, Bohner M, Heini P, et al. Performance of vertebral cancellous bone augmented with compliant PMMA under dynamic loads. Acta Biomaterialia. 2008; 4: 1688-1693.

29. Boger A, Wheeler K, Montali A, et al. NMP-modified PMMA bone cement with adapted mechanical and hardening properties for the use in cancellous bone augmentation. Journal of Biomedical Materials Research Part B: Applied Biomaterials. 2009; 90B: 760-766.

30. Beck SandBoger A. Evaluation of the particle release of porous PMMA cements during curing. Acta Biomater. 2009; 5: 2503-2507.

31. Seniha Güner F, Yagci YandTuncer Erciyes A. Polymers from triglyceride oils. Progress in Polymer Science. 2006; 31: 633-670.

32. Vázquez B, Deb S, Bonfield W, et al. Characterization of new acrylic bone cements prepared with oleic acid derivatives. Journal of Biomedical Materials Research. 2002; 63: 88-97.

33. Lam WM, Pan HB, Fong MK, et al. In Vitro characterization of low modulus linoleic acid coated strontium-substituted hydroxyapatite containing PMMA bone cement. Journal of Biomedical Materials Research Part B: Applied Biomaterials. 2011; 96B: 76-83.

34. Lopez A, Hoess A, Thersleff T, et al. Low-modulus PMMA bone cement modified with castor oil. Biomed Mater Eng. 2011; 21: 323-332.

35. Webb JCandSpencer RF. The role of polymethylmethacrylate bone cement in modern orthopaedic surgery. J Bone Joint Surg Br. 2007; 89: 851-857.

36. Zheng CJ, Yoo JS, Lee TG, et al. Fatty acid synthesis is a target for antibacterial activity of unsaturated fatty acids. FEBS Lett. 2005; 579: 5157-5162.

37. Valera MC, Maekawa LE, Chung A, et al. Effectiveness of castor oil extract on Escherichia coli and its endotoxins in root canals. Gen Dent. 2012; 60: e204209.

38. ISO 5833 Implants for surgery - Acrylic resin cements. The International Organization for Standardization; 2002. 
39. Baleani M, Persson C, Zolezzi C, et al. Biological and biomechanical effects of vancomycin and meropenem in acrylic bone cement. $J$ Arthroplasty. 2008; 23: 1232-1238

40. Bertazzoni Minelli E, Benini A, Samaila E, et al. Antimicrobial activity of gentamicin and vancomycin combination in joint fluids after antibiotic-loaded cement spacer implantation in two-stage revision surgery. J Chemother. 2014: 1973947813 Y0000000157.

41. ISO 10993-5: Biological evaluation of medical devices - Part 5: Tests for in vitro cytotoxicity.: International Organization for Standardization; 2009.

42. Guo JandSchork FJ. Hybrid miniemulsion polymerization of acrylate/oil and acrylate/fatty acid systems. Macromolecular Reaction Engineering. 2008; 2: 265-276.

43. Lam WM, Pan HB, Fong MK, et al. In Vitro characterization of low modulus linoleic acid coated strontium-substituted hydroxyapatite containing PMMA bone cement. J Biomed Mater Res B Appl Biomater. 2011; 96: 76-83.

44. Li S, Chien SandBranemark PI. Heat shock-induced necrosis and apoptosis in osteoblasts. J Orthop Res. 1999; 17: 891-899.

45. Berman AT, Reid JS, Yanicko DR, Jr., et al. Thermally induced bone necrosis in rabbits. Relation to implant failure in humans. Clin Orthop Relat Res. 1984: 284-292.

46. Narasimhan B, Mourya VKandDhake AS. QSAR studies of antibacterial ricinoleic acid derivatives. Pharmaceutical Chemistry Journal. 2007; 41: 133139.

47. Gough JEandDownes S. Osteoblast cell death on methacrylate polymers involves apoptosis. J Biomed Mater Res. 2001; 57: 497-505.

48. Geurts JA, Janssen DM, Kessels AG, et al. Good results in postoperative and hematogenous deep infections of 89 stable total hip and knee replacements with retention of prosthesis and local antibiotics. Acta Orthop. 2013; 84: 509-516. 
Table 1. Mechanical properties ( $E$ : Young's modulus; $\sigma_{c}$ : compressive strength) of unmodified bone cements Simplex P and Osteopal V

\begin{tabular}{ccc}
\cline { 2 - 3 } & Simplex $\mathbf{P}(\mathbf{n}=\mathbf{1 0})$ & Osteopal $\mathbf{V}(\mathbf{n}=\mathbf{6})$ \\
\hline $\boldsymbol{E}[\mathrm{MPa}]$ & $1632 \pm 39$ & $1524 \pm 66$ \\
$\boldsymbol{\sigma}_{c}[\mathrm{MPa}]$ & $81.6 \pm 2.6$ & $77.0 \pm 1.6$ \\
\hline
\end{tabular}


Table 2. Mechanical properties ( $E$ : Young's modulus; $\sigma_{c}$ : compressive strength) of fatty acid modified bone cements at different concentrations (wt $\%$ of total cement).

\begin{tabular}{|c|c|c|c|c|c|c|c|}
\hline & Ricinoleic acid [wt\%] & $3.35(n=6)$ & $6.60(n=6)$ & $9.75(n=4)$ & $12.80(n=6)$ & $15.77(n=4)$ & - \\
\hline$E$ [MPa] & Simplex P & $1504 \pm 179$ & $1294 \pm 39$ & $1143 \pm 36$ & $507 \pm 149$ & $313 \pm 70$ & - \\
\hline \multirow[t]{2}{*}{$\sigma_{c}[\mathrm{MPa}]$} & & $63.7 \pm 16.6$ & $40.6 \pm 1.3$ & $29.4 \pm 0.6$ & $12.0 \pm 1.0$ & $7.1 \pm 0.2$ & - \\
\hline & Ricinoleic acid [wt\%] & $3.61(n=5)$ & $7.10(n=8)$ & $10.48(n=4)$ & $13.76(n=6)$ & - & - \\
\hline$E$ [MPa] & Osteopal V & $1393 \pm 41.4$ & $1047 \pm 64$ & $798 \pm 45$ & $424 \pm 40$ & - & - \\
\hline \multirow[t]{2}{*}{$\sigma_{c}[\mathrm{MPa}]$} & & $49.6 \pm 2.2$ & $31.8 \pm 1.0$ & $21.0 \pm 3.7$ & $10.8 \pm 1.2$ & - & - \\
\hline & Methyl linoleate [wt $\%$ ] & $0.68(\mathrm{n}=6)$ & $1.02(n=6)$ & $1.35(n=6)$ & $1.69(n=6)$ & $2.02(n=6)$ & $2.69(n=6)$ \\
\hline$E$ [MPa] & Simplex P & $1477 \pm 209$ & $1367 \pm 68$ & $1131 \pm 27$ & $947 \pm 41$ & $502 \pm 27$ & $307 \pm 6$ \\
\hline \multirow[t]{2}{*}{$\sigma_{c}[\mathrm{MPa}]$} & & $63.6 \pm 1.5$ & $49.2 \pm 2.0$ & $31.7 \pm 0.5$ & $23.1 \pm 0.3$ & $12.6 \pm 0.2$ & $6.6 \pm 0.1$ \\
\hline & Methyl linoleate [wt\%] & $0.37(n=6)$ & $0.55(n=5)$ & $0.91(\mathrm{n}=5)$ & - & - & - \\
\hline$E$ [MPa] & Osteopal V & $1563 \pm 127$ & $1143 \pm 66$ & $456 \pm 16$ & - & - & - \\
\hline \multirow[t]{2}{*}{$\sigma_{c}[\mathrm{MPa}]$} & & $54.1 \pm 1.3$ & $33.8 \pm 1.6$ & $9.8 \pm 0.3$ & - & - & - \\
\hline & Linoleic acid [wt\%] & $0.34(n=6)$ & $0.68(n=6)$ & $1.02(n=6)$ & $1.36(n=6)$ & $1.69(n=6)$ & $2.02(n=6)$ \\
\hline$E$ [MPa] & Simplex P & $1565 \pm 40$ & $1488 \pm 52$ & $1190 \pm 71$ & $768 \pm 19$ & $424 \pm 17$ & $249 \pm 10$ \\
\hline \multirow[t]{2}{*}{$\sigma_{c}[\mathrm{MPa}]$} & & $70.2 \pm 2.8$ & $60.3 \pm 2.2$ & $61.8 \pm 0.9$ & $18.6 \pm 0.3$ & $10.5 \pm 0.3$ & $5.9 \pm 0.2$ \\
\hline & Linoleic acid [wt\%] & $0.50(n=6)$ & $0.75(n=6)$ & $1.00(n=6)$ & - & - & - \\
\hline$E$ [MPa] & Osteopal V & $1486 \pm 51$ & $1047 \pm 61$ & $337 \pm 53$ & - & - & - \\
\hline$\sigma_{c}[\mathrm{MPa}]$ & & $53.0 \pm 1.6$ & $27.2 \pm 0.7$ & $11.4 \pm 0.9$ & - & - & - \\
\hline
\end{tabular}


Table 3. Mechanical properties ( $E$ : Young's modulus; $\sigma_{c}$ : compressive strength) of triglyceride oil modified bone cements at different concentrations (wt $\%$ of total cement).

\begin{tabular}{|c|c|c|c|c|c|c|c|c|}
\hline & Linseed oil [wt\%] & $3.35(n=6)$ & $6.60(n=3)$ & $9.75(n=6)$ & $10.99(n=5)$ & $12.81(n=6)$ & - & - \\
\hline$E$ [MPa] & Simplex P & $1291 \pm 53$ & $1309 \pm 35$ & $1103 \pm 175$ & $606 \pm 91$ & $402 \pm 124$ & - & - \\
\hline \multirow[t]{2}{*}{$\sigma_{c}$ [MPa] } & & $46.7 \pm 1.4$ & $43.4 \pm 2.4$ & $34.6 \pm 6.1$ & $16.6 \pm 2.4$ & $10.1 \pm 2.0$ & - & - \\
\hline & Linseed oil [wt\%] & $0.73(n=6)$ & $1.82(\mathrm{n}=6)$ & $2.36(\mathrm{n}=5)$ & - & - & - & - \\
\hline$E$ [MPa] & Osteopal V & $705 \pm 76$ & $631 \pm 36$ & $408 \pm 31$ & - & - & - & - \\
\hline \multirow[t]{2}{*}{$\sigma_{c}[\mathrm{MPa}]$} & & $17.9 \pm 2.2$ & $14.7 \pm 1.0$ & $10.0 \pm 0.4$ & - & - & - & - \\
\hline & Tung oil [wt\%] & $0.17(n=6)$ & $0.31(n=6)$ & $0.68(n=6)$ & $1.35(n=6)$ & - & - & - \\
\hline$E$ [MPa] & Simplex P & $1460 \pm 72$ & $1473 \pm 27$ & $1200 \pm 30$ & $310 \pm 33$ & - & - & - \\
\hline \multirow[t]{2}{*}{$\sigma_{c}[\mathrm{MPa}]$} & & $68.7 \pm 1.9$ & $61.4 \pm 3.9$ & $37.7 \pm 0.9$ & $8.1 \pm 0.8$ & - & - & - \\
\hline & Tung oil [wt\%] & $0.18(n=6)$ & $0.37(n=6)$ & - & - & - & - & - \\
\hline$E$ [MPa] & Osteopal V & $1505 \pm 66$ & $16 \pm 4$ & - & - & - & - & - \\
\hline \multirow[t]{2}{*}{$\sigma_{c}[\mathrm{MPa}]$} & & $46.6 \pm 1.6$ & $0.6 \pm 0.1$ & - & - & - & - & - \\
\hline & Castor oil [wt\%] & $3.35(n=6)$ & $8.18(n=6)$ & $9.74(\mathrm{n}=5)$ & $11.27(n=4)$ & $12.79(n=6)$ & $14.57(n=6)$ & $16.90(n=6)$ \\
\hline$E$ [MPa] & Simplex P & $1594 \pm 36$ & $1352 \pm 38$ & $1137 \pm 27$ & $861 \pm 60$ & $644 \pm 51$ & $558 \pm 51$ & $217 \pm 36$ \\
\hline \multirow[t]{2}{*}{$\sigma_{c}[\mathrm{MPa}]$} & & $77.9 \pm 2.5$ & $46.1 \pm 5.1$ & $38.7 \pm 1.3$ & $26.8 \pm 1.2$ & $16.8 \pm 1.1$ & $13.0 \pm 1.1$ & $4.1 \pm 0.3$ \\
\hline & Castor oil [wt\%] & $7.78(n=6)$ & $10.47(n=5)$ & $12.77(n=8)$ & $13.73(n=5)$ & - & - & - \\
\hline$E$ [MPa] & Osteopal V & $1190 \pm 35$ & $786 \pm 87$ & $429 \pm 71$ & $361 \pm 38$ & - & - & - \\
\hline$\sigma_{c}$ [MPa] & & $38.4 \pm 1.5$ & $23.8 \pm 2.3$ & $12.7 \pm 2.3$ & $10.3 \pm 1.1$ & - & - & - \\
\hline
\end{tabular}


Table 4. Mechanical properties (E: Young's modulus; $\sigma_{c}$ : compressive strength) of the selected cements, with and without Vancomycin.

\begin{tabular}{ccccc}
\hline $\begin{array}{c}\text { Without } \\
\text { Vancomycin }\end{array}$ & OP $(\mathbf{n}=\mathbf{6})$ & OP-1.0\%LA $(\mathbf{n}=\mathbf{8})$ & OP-12.6\%RA $(\mathbf{n}=\mathbf{6})$ & OP-12.3\%CO $(\mathbf{n}=\mathbf{6})$ \\
$\begin{array}{c}\boldsymbol{E}[\mathrm{MPa}] \\
\boldsymbol{\sigma}_{c}[\mathrm{MPa}]\end{array}$ & $1524 \pm 66$ & $337 \pm 53$ & $418 \pm 21$ & $432 \pm 59$ \\
\hline $\begin{array}{c}\text { With } \\
\text { Vancomycin }\end{array}$ & OP-VA $(\mathbf{n}=7)$ & OP-1.0\%LA-VA $(\mathbf{n}=7)$ & OP-12.6\%RA-VA (n=6) & OP-12.3\%CO-VA (n=6) \\
$\boldsymbol{E}[\mathrm{MPa}]$ & $1653 \pm 57$ & $427 \pm 37$ & $406 \pm 41$ & $417 \pm 58$ \\
$\boldsymbol{\sigma}_{c}[\mathrm{MPa}]$ & $77.9 \pm 1.7$ & $12.4 \pm 0.4$ & $13.9 \pm 1.3$ & $14.5 \pm 3.8$ \\
\hline
\end{tabular}

Table 5. Effect of additives on the setting time, maximum polymerization temperature and glass transition temperature of the selected cements.

\begin{tabular}{cccc}
\hline Cement & $\begin{array}{c}\text { Setting time } \\
{[\mathrm{min}]}\end{array}$ & $\begin{array}{c}\text { Peak temperature } \\
{\left[{ }^{\circ} \mathrm{C}\right]}\end{array}$ & $\mathrm{T}_{\mathrm{g}}\left({ }^{\circ} \mathrm{C}\right)$ \\
\hline OP & $\mathbf{2 1 . 1} \pm \mathbf{1 . 1}$ & $\mathbf{6 3 . 5} \pm \mathbf{2 . 6}$ & 98.4 \\
OP-1.0\%LA & $\mathbf{1 9 . 7} \pm \mathbf{0 . 9}$ & $\mathbf{3 5 . 3} \pm \mathbf{0 . 4}$ & 86.4 \\
OP-12.6\%RA & $\mathbf{1 3 . 7} \pm \mathbf{0 . 7}$ & $\mathbf{3 5 . 0} \pm \mathbf{0 . 8}$ & 57.7 \\
OP-12.3\%CO & $\mathbf{1 5 . 6} \pm \mathbf{0 . 1}$ & $\mathbf{3 4 . 2} \pm \mathbf{2 . 7}$ & 74.0 \\
\hline
\end{tabular}



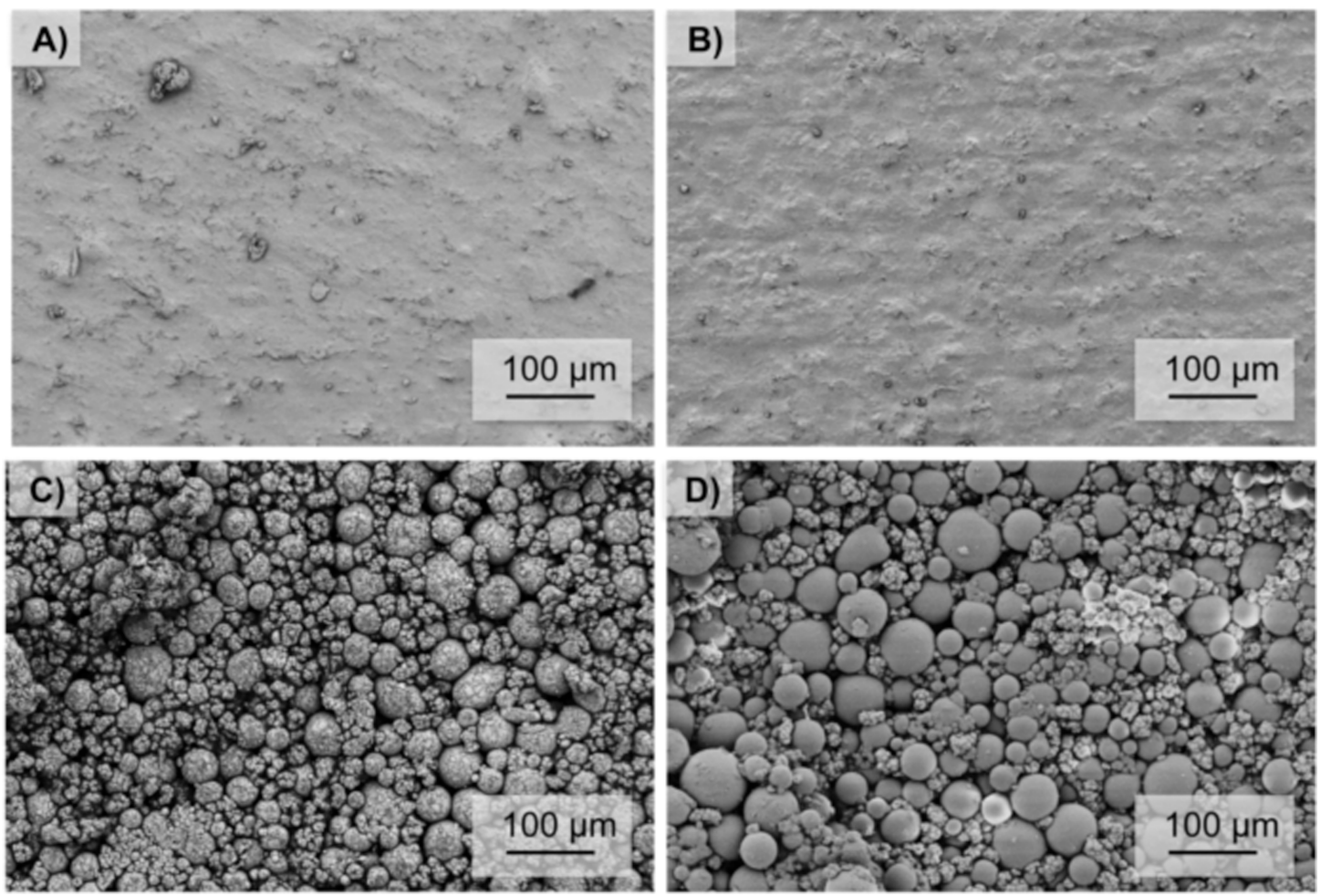

Figure 1. Representative scanning electron micrographs of A) unmodified OP; B) OP-1.0\%LA; C) OP-12.6\%RA; D) OP-12.3\%CO.
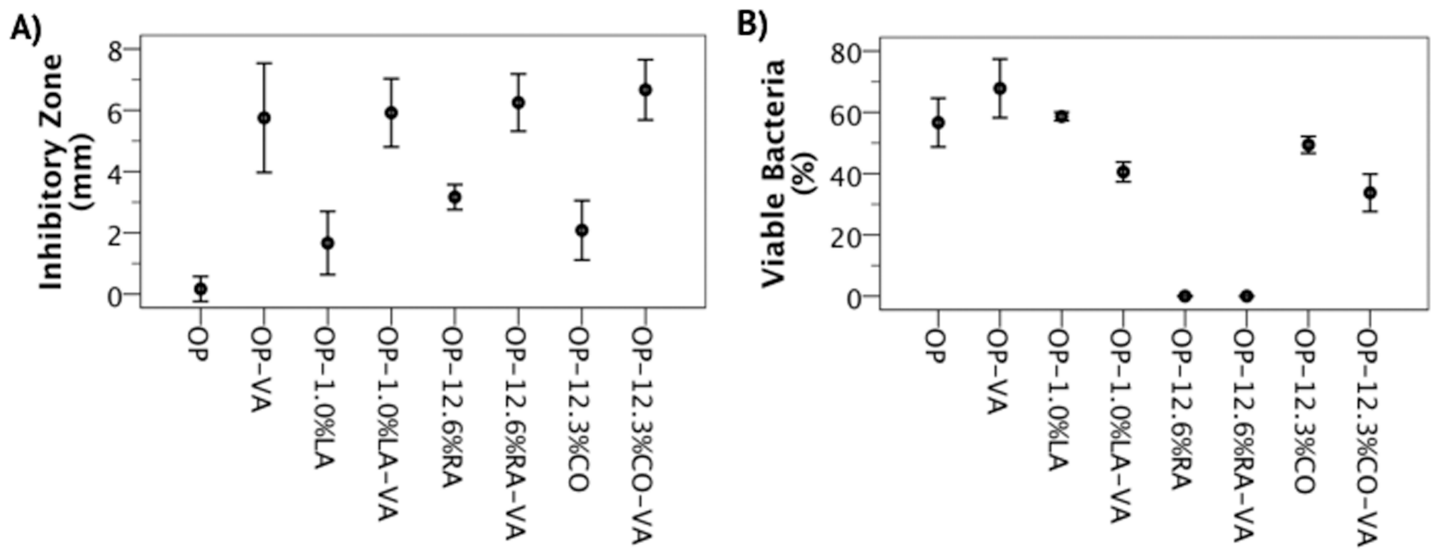

Figure 2. The resulting inhibitory zone from the agar diffusion test (A) and the \% viable bacteria after exposure to extracts of the evaluated cements (B). Results are presented as average values \pm standard deviation, for six replicates per group for the results in (A) and 4 replicates per group for the results in (B), except for OP$12.3 \% \mathrm{CO}$, where only 3 data points were available for $(\mathrm{B})$. 